PROCEEDINGS OF THE

AMERICAN MATHEMATICAL SOCIETY

Volume 129, Number 1, Pages 89-95

S 0002-9939(00)05860-3

Article electronically published on August 17, 2000

\title{
THE BLOW-UP FOR WEAKLY COUPLED REACTION-DIFFUSION SYSTEMS
}

\author{
LIWEN WANG
}

(Communicated by David S. Tartakoff)

\begin{abstract}
In this paper we consider a weakly coupled parabolic system with nonnegative exponents in the forcing functions. We find the conditions which result in blow-up in finite time. Also, we obtain the blow-up rate.
\end{abstract}

\section{INTRODUCTION}

In this paper, we consider the following system:

$$
\begin{array}{lll}
u_{t}-\Delta u=u^{p_{1}} v^{p_{2}} & \text { for } x \in \Omega, & t>0, \\
v_{t}-\Delta v=u^{p_{3}} v^{p_{4}} & \text { for } x \in \Omega, & t>0,
\end{array}
$$

with the initial and boundary conditions

$$
\begin{gathered}
u(x, 0)=u_{0}(x), v(x, 0)=v_{0}(x), \\
\left.u\right|_{\partial \Omega}=0,\left.v\right|_{\partial \Omega}=0,
\end{gathered}
$$

where $\Omega$ is a bounded domain in $R^{N} . p_{i}$ for $i=1,2,3,4$, are nonnegative real numbers. We assume that $p_{2}-p_{4}+1 \geq p_{3}-p_{1}+1>0$.

The study of (1.1), (1.2) is of great interest due to its application. The above models arise in the chemical reaction processes. The density and temperature are governed by a coupled system of reaction diffusion equations in the form of (1.1), (1.2).

In the past years, several authors have studied such a problem. Galaktionov, Kurdyumov and Samarskii obtained the first blow-up results for the semilinear and quasilinear systems of type (1.1), (1.2) in [6], [7]; in particular they established a sufficient condition of global blow-up for general quasilinear systems. Deng [1] studied the blow-up rate for systems like (1.1), (1.2) with $p_{1}=p_{4}=0$.

In [13, 14] the authors considered a similar system with different source terms

$$
\begin{aligned}
& u_{t}-\Delta u=u f_{1}(v) \quad \text { for } x \in \Omega, \quad t>0 \text {, } \\
& v_{t}-\Delta v=v f_{2}(u) \quad \text { for } x \in \Omega, \quad t>0 \text {, }
\end{aligned}
$$

with initial boundary condition. This system can be applied to the special case of (1.1), (1.2) with $p_{1}=p_{4}=1$ and $1<p_{2}, p_{3}<2$. The authors have proved

Received by the editors March 7, 1999.

2000 Mathematics Subject Classification. Primary 35K55, 35K57, 35K40.

Key words and phrases. Blow-up, weakly coupled reaction-diffusion system.

(C)2000 American Mathematical Society 
the existence of a blow-up solution and blow-up rate in the case of a single-point blow-up for the initial boundary value problem.

Some early results can also be seen in [4, [5], 11]. In [8], 10, the authors considered the blow-up problem of linear equation with nonlinear boundary condition, and obtained the blow-up rate.

My motivation to study (1.1), (1.2) comes from the results of [3] (in [2] Escobedo and Herrero considered the special case $\left.p_{1}=p_{4}=0\right)$. The authors considered this system with the Cauchy problem and obtained Fujita-type global existence and global nonexistence theorems for (1.1), (1.2) analogous to the classical result of Fujita and others for the initial-value problem for $u_{t}-\Delta u=u^{p}, u(x, 0)=$ $u_{0}(x) \geq 0$. Similar results can be seen in [12].

In this paper we find the necessary and sufficient conditions which result in blow-up and obtain some results about the blow-up rate for (1.1), (1.2).

The paper is organized as follows: In section 2 we prove blow-up and global existence results and in section 3 we present the blow-up rate results.

\section{Blow-UP AND GLOBAL EXISTEnCE}

In this section we consider the system (1.1), (1.2) with the initial and boundary conditions

$$
\begin{aligned}
u(x, 0) & =u_{0}(x), v(x, 0)=v_{0}(x), \\
\left.u\right|_{\partial \Omega} & =1,\left.v\right|_{\partial \Omega}=1
\end{aligned}
$$

where $u_{0}(x)$ and $v_{0}(x)$ are nonnegative functions.

Initially we develop a lemma which will be needed to prove our main results.

Lemma 2.1. Assume that $C_{0} u_{0}(x) \geq v_{0}(x)^{\gamma_{0}}$, where $\gamma_{0}=\frac{p_{2}-p_{4}+1}{p_{3}-p_{1}+1}$ and $C_{0} \geq$ $\left(\gamma_{0}\right)^{\frac{1}{p_{3}-p_{1}+1}}$. Let $(u, v)$ be a nonnegative solution of the system (1.1), (1.2) with (2.1), (2.2). Then we have

$$
C_{0} u(x, t) \geq v(x, t)^{\gamma_{0}} .
$$

Proof. Let $J=u^{p_{3}-p_{1}+1}-C v^{p_{2}-p_{4}+1}$. Then

$$
\begin{aligned}
J_{t}- & \Delta J=\left[\left(p_{3}-p_{1}+1\right)-C\left(p_{2}-p_{4}+1\right)\right] u^{p_{3}} v^{p_{2}} \\
- & \left(p_{3}-p_{1}+1\right)\left(p_{3}-p_{1}\right) u^{p_{3}-p_{1}-1}|\nabla u|^{2} \\
& +C\left(p_{2}-p_{4}+1\right)\left(p_{2}-p_{4}\right) v^{p_{2}-p_{4}-1}|\nabla v|^{2} .
\end{aligned}
$$

If we let $0<C<\frac{p_{3}-p_{1}+1}{p_{2}-p_{4}+1}$, we have

$$
\left[\left(p_{3}-p_{1}+1\right)-C\left(p_{2}-p_{4}+1\right)\right] u^{p_{3}} v^{p_{2}} \geq 0 .
$$

Using the definition of $J$, we have

$$
\left(p_{3}-p_{1}+1\right) u^{p_{3}-p_{1}} \nabla u=\nabla J+C\left(p_{2}-p_{4}+1\right) v^{p_{2}-p_{4}}|\nabla u|^{2}=\nabla v ;
$$

squaring both sides, we have

$$
|\nabla u|^{2}=\left[\frac{C\left(p_{2}-p_{4}+1\right)}{p_{3}-p_{1}+1}\right]^{2} v^{2\left(p_{2}-p_{4}\right)} u^{-2\left(p_{3}-p_{1}\right)}|\nabla v|^{2}+b_{1} \nabla J,
$$

where $b_{1}$ is a bounded function. Since

$$
C v^{p_{2}-p_{4}+1}=u^{p_{3}-p_{1}+1}-J
$$


we have

$$
\begin{aligned}
\left(p_{3}-p_{1}+1\right)\left(p_{3}-p_{1}\right) u^{p_{3}-p_{1}-1}|\nabla u|^{2} & \\
& =\frac{\left(p_{3}-p_{1}\right)\left(p_{2}-p_{4}+1\right)^{2} C}{\left(p_{3}-p_{1}+1\right)} v^{p_{2}-p_{4}-1}|\nabla v|^{2}+b_{2} J \\
& \leq C\left(p_{2}-p_{4}+1\right)\left(p_{2}-p_{4}\right) v^{p_{2}-p_{4}-1}|\nabla v|^{2}+b_{2} J
\end{aligned}
$$

where $b_{2}$ is a bounded function. This proves

$$
J_{t}-\Delta J-b_{1} \nabla J-b_{2} J \geq 0
$$

Letting $C \leq\left(C_{0}\right)^{p_{3}-p_{1}+1}$, we have

$$
\begin{aligned}
\left.J\right|_{\partial \Omega} & \geq 0 \\
\left.J\right|_{t=0} & \geq 0
\end{aligned}
$$

By the maximum principle, we have

$$
J(x, t) \geq 0 \quad \text { for } t>0 \text { and } x \in \Omega .
$$

This means $u^{p_{3}-p_{1}+1} \geq C v^{p_{2}-p_{4}+1}$. This is

$$
C_{0} u(x, t) \geq v(x, t)^{\gamma_{0}}
$$

Therefore this completes the proof.

Remark. The assumption $C_{0} u_{0}(x) \geq v_{0}(x)^{\gamma_{0}}$ is obviously true when $u_{0}(x)>0$ for $x \in \bar{\Omega}$. For in this case we can choose a large enough constant $C_{0}$ such that the assumption holds. If $u_{0}(x) \geq 0$, we can choose some $t_{0}>0$ such that $u\left(x, t_{0}\right)>0$ for $x \in \bar{\Omega}$.

We introduce some notation to develop our next results:

$$
P=\left(\begin{array}{ll}
p_{1} & p_{2} \\
p_{3} & p_{4}
\end{array}\right), \quad I=\left(\begin{array}{ll}
1 & 0 \\
0 & 1
\end{array}\right)
$$

and $|P|=\operatorname{det} P$.

Theorem 2.2. If $|P-I| \geq 0$, then the solution (u,v) of the system (1.1), (1.2) with (2.1), (2.2) is global for any bounded initial data.

Proof. Using inequality (2.3) and the equation (1.1), we have

$$
u_{t}-\Delta u \leq C^{\prime} u^{p_{1}} u^{p_{2} \gamma_{0}^{-1}}=C^{\prime} u^{\left[-|P-I| /\left(p_{2}-p_{4}+1\right)\right]+1} .
$$

Since $|P-I| \geq 0, u$ is the lower solution of the following:

$$
\begin{aligned}
& w_{t}-\Delta w=w^{\lambda} \quad \text { for } t>0, x \in \Omega, \\
& \left.w\right|_{\partial \Omega}=0, \\
& \left.w\right|_{t=0}=u_{0}(x)
\end{aligned}
$$

where $0<\lambda=\left[-|P-I| /\left(p_{2}-p_{4}+1\right)\right]+1 \leq 1, w$ exists globally, so $u$ globally exists. By (2.3) so does $v$.

Theorem 2.3. If $|P-I|<0$, and $\Delta u_{0}+u_{0}^{p_{1}} v_{0}^{p_{2}} \geq 0, \Delta v_{0}+u_{0}^{p_{3}} v_{0}^{p_{4}} \geq 0$, then the solution of system (1.1), (1.2) with (2.1), (2.2) blows up. 
Proof. Using inequality (2.3) and the equation (1.2), we have

$$
v_{t}-\Delta v \geq C v^{1+\alpha} \quad \text { for } x \in \Omega, \quad t>0,
$$

where $\alpha=\frac{-|P-I|}{p_{3}-p_{1}+1}>0$ and $C$ is a constant. It is easy to obtain that $v$ blows up. Since (2.3), so does $u$.

Remark. The system (1.1), (1.2) with (1.3), (1.4) has the same nonglobal results as in section 2 by constructing a lower blow-up solution $\left(w_{1}, w_{2}\right)$ in smaller domain $\Omega^{\prime} \subset \Omega$ as follows:

$$
\begin{aligned}
& w_{1 t}-\Delta w_{1}=w_{1}^{p_{1}} w_{2}^{p_{2}} \quad \text { for } x \in \Omega^{\prime}, \quad t>0, \\
& w_{2 t}-\Delta w_{2}=w_{1}^{p_{3}} w_{2}^{p_{4}} \quad \text { for } x \in \Omega^{\prime}, \quad t>0,
\end{aligned}
$$

with the initial and boundary conditions

$$
\begin{aligned}
w_{1}(x, 0) & =u\left(x, t_{0}\right), w_{2}(x, 0)=v\left(x, t_{0}\right), \\
\left.w_{1}\right|_{\partial \Omega^{\prime}} & =\left.u\left(x, t_{0}\right)\right|_{\partial \Omega^{\prime}}>0,\left.w_{2}\right|_{\partial \Omega^{\prime}}=\left.v\left(x, t_{0}\right)\right|_{\partial \Omega^{\prime}}>0 .
\end{aligned}
$$

It is similar for global existence.

\section{BLOW-UP RATE}

In this section, we assume that $p_{i} \geq 1$ for $i=1,2,3,4$ are nonnegative real numbers. Also $u_{0}(x)$ and $v_{0}(x)$ are nonnegative functions, increase in $\left(0, \frac{l}{2}\right)$ and decrease in $\left(\frac{l}{2}, l\right)$.

Lemma 3.1. Assume that $C_{0} u_{0}(x) \geq v_{0}(x)^{\gamma_{0}}$, where $\gamma_{0}=\frac{p_{2}-p_{4}+1}{p_{3}-p_{1}+1}$ and $C_{0} \geq$ $\left(\gamma_{0}\right)^{\frac{1}{p_{3}-p_{1}+1}}$. Let $(u, v)$ be a nonnegative solution of the system (1.1), (1.2). Then we have

$$
C_{0}(u(x, t)+1) \geq v(x, t)^{\gamma_{0}} .
$$

The proof is similar to that of Lemma 2.1.

Theorem 3.2. For the solutions $u(x, t), v(x, t)$ of system (1.1), (1.2), let $U(t)=$ $\max _{x \in \Omega} u(x, t)$ and $V(t)=\max _{x \in \Omega} v(x, t)$. Then the functions are Lipschitz continuous and

$$
\begin{array}{ll}
U_{t} \leq U^{p_{1}} V^{p_{2}} & \text { for } t>0 \text { a.e. } \\
V_{t} \leq U^{p_{3}} V^{p_{4}} & \text { for } t>0 \quad \text { a.e. }
\end{array}
$$

The proof is very similar to that of Theorem 4.5 in [5], hence we omit it.

Theorem 3.3. For the solutions $u(x, t), v(x, t)$ of system (1.1), (1.2), and initial functions $u_{0}(x, t)$ and $v_{0}(x, t)$ are symmetric for $x=\frac{l}{2}$. Further, set $u_{0}(x)$ and $v_{0}(x)$ to be increasing functions for $0<x<\frac{l}{2}$. Also there exists a constant $0<$ $\delta<1$ such that $\Delta u_{0}+(1-\delta) u_{0}^{p_{1}} v_{0}^{p_{2}} \geq 0, \Delta v_{0}+(1-\delta) u_{0}^{p_{3}} v_{0}^{p_{4}} \geq 0$. Then

$$
\begin{aligned}
& u_{t} \geq \delta u^{p_{1}} v^{p_{2}} \quad \text { for } x \in \Omega, \quad t>0, \\
& v_{t} \geq \delta u^{p_{3}} v^{p_{4}} \quad \text { for } x \in \Omega, \quad t>0 \text {. }
\end{aligned}
$$


Proof. Since $u_{0}(x)$ and $v_{0}(x)$ increase for $0<x<\frac{l}{2}$, we have that $u(x, t)$ and $v(x, t)$ increase for $0<x<\frac{l}{2}$ (see [4, 5]). Letting $F(x, t)=u_{t}+\frac{\delta}{1-\delta} u_{x x}$ and $G(x, t)=v_{t}+\frac{\delta}{1-\delta} v_{x x}$, we have

$$
\begin{aligned}
F_{t}-F_{x x} & \geq p_{1} u^{p_{1}-1} v^{p_{2}} F+p_{2} u^{p_{1}} v^{p_{2}-1} G, \\
G_{t}-G_{x x} & \geq p_{3} u^{p_{3}-1} v^{p_{4}} F+p_{4} u^{p_{3}} v^{p_{4}-1} G,
\end{aligned}
$$

and

$$
\begin{gathered}
\left.F(x, t)\right|_{x=0, l}=0,\left.G(x, t)\right|_{x=0, l}=0, \\
F(x, 0) \geq 0, G(x, 0) \geq 0 .
\end{gathered}
$$

By the maximum principle, one can see that both $F$ and $G$ are nonnegative in $(0, l) \times(0, T)$. From equation $(1.1),(1.2)$, we have

$$
\begin{aligned}
& \frac{1}{\delta} u_{t} \geq u^{p_{1}} v^{p_{2}}, \\
& \frac{1}{\delta} v_{t} \geq u^{p_{3}} v^{p_{4}} .
\end{aligned}
$$

This completes the proof.

Theorem 3.4. If $|P-I|<0, \Delta u_{0}+(1-\delta) u_{0}^{p_{1}} v_{0}^{p_{2}} \geq 0, \Delta v_{0}+(1-\delta) u_{0}^{p_{3}} v_{0}^{p_{4}} \geq$ $0, u(x, t)$ and $v(x, t)$ are the solutions of the system (1.1), (1.2), and $T$ is the blowup time, then

$$
\begin{aligned}
& C_{1}(T-t)^{\alpha_{1}} \leq \max _{x \in \Omega} u(x, t), \\
& \max _{x \in \Omega} v(x, t) \leq C_{2}(T-t)^{\alpha_{2}},
\end{aligned}
$$

where $\alpha_{1}=\frac{p_{2}-p_{4}+1}{|P-I|}, \quad \alpha_{2}=\frac{p_{3}-p_{1}+1}{|P-I|}$ and $C_{i}$ are positive constants.

Proof. Since $U(t) \rightarrow \infty$ as $t \rightarrow T$, there exists a $t_{0}$ such that $U(t)>1$ for any $t>t_{0}$. Hence we have

$$
2 C_{0} U(t) \geq V(t)^{\gamma_{0}} .
$$

Letting $(x(t), t)$ be the points at which $v(x, t)$ attains its maximum, for any $t_{2}>$ $t_{1}>t_{0}$, we have

$$
\frac{V\left(t_{2}\right)-V\left(t_{1}\right)}{t_{2}-t_{1}} \geq \frac{v\left(x\left(t_{1}\right), t_{2}\right)-v\left(x\left(t_{1}\right), t_{1}\right)}{t_{2}-t_{1}}=v_{t}\left(t_{1}\right)+o(1) .
$$

From (3.5), (3.8) and (3.9), we have

$$
V_{t} \geq C V(t)^{1+\alpha}
$$

where $\alpha=\frac{-|P-I|}{p_{3}-p_{1}+1}$ and $C$ is a constant. Integrating (3.10) from $t$ to $T$ yields

$$
V(t) \leq C_{2}(T-t)^{\alpha_{2}} \text {. }
$$

Combining (3.8) and (3.2), we have

$$
U_{t} \leq C U(t)^{1+\alpha}
$$

where $\alpha=\frac{-|P-I|}{p_{2}-p_{4}+1}$ and $C$ is a constant. Integrating (3.12) from $t$ to $T$, we obtain

$$
U(t) \geq C_{1}(T-t)^{\alpha_{1}} .
$$

This completes the proof. 
Theorem 3.5. Assume the conditions of Theorem 3.4 are true and

$$
\max _{x \in \Omega} u(x, t) \leq C_{3}(T-t)^{\alpha_{1}}
$$

then

$$
\max _{x \in \Omega} v(x, t) \geq C_{4}(T-t)^{\alpha_{2}} .
$$

Proof. We claim that $V(t)(T-t)^{-\alpha_{1}}$ is bounded from below by a positive constant $C_{4}$. Otherwise, we assume that $\operatorname{maxinf}_{t \rightarrow T} V(t)(T-t)^{-\alpha_{1}}=0$. Then there exist a sequence $\left\{t_{k}\right\} \in(0, T)$ with $t_{k} \rightarrow T$ and a sequence $\left\{\epsilon_{k}\right\}$ with $\epsilon_{k} \rightarrow 0$ such that

$$
V(t)(T-t)^{-\alpha_{1}} \leq \epsilon_{k} \quad \text { for any } k=1,2,3, \cdots
$$

Now we choose a positive integer $m$ such that $C_{3}(m+1)^{\alpha_{2}} \leq \frac{C_{1}}{2}$. For such a choice of $m$, when $t_{k}$ is close to $T$ we can select a corresponding sequence $\left\{\tau_{k}\right\}$ such that $T-\tau_{k}=(m+1)\left(T-t_{k}\right)$. Now using (3.2), (3.14) and (3.11), we would obtain that

$$
\begin{aligned}
U\left(t_{k}\right) & \leq U\left(\tau_{k}\right)+\int_{\tau_{k}}^{t_{k}} U^{p_{1}}(s) V^{p_{2}}(s) d s \\
& \leq C_{3}\left(T-\tau_{k}\right)^{\alpha_{1}}+\left[C_{3}\left(T-t_{k}\right)^{\alpha_{1}}\right]^{p_{1}} V^{p_{2}}\left(t_{k}\right)\left(t_{k}-\tau_{k}\right) \\
& \leq C_{3}(m+1)^{\alpha_{1}}\left(T-t_{k}\right)^{\alpha_{2}}+C_{3}^{p_{2}}\left(T-t_{k}\right)^{\alpha_{1} p_{2}+1} V^{p_{2}}\left(t_{k}\right) \\
& \leq \frac{C_{1}}{2}\left(T-t_{k}\right)^{\alpha_{1}}+C_{3}^{p_{2}}\left(T-t_{k}\right)^{\alpha_{1} p_{2}+1-\alpha_{1} p_{1}} \epsilon^{p_{1}} \\
& \leq \frac{C_{1}}{2}\left(T-t_{k}\right)^{\alpha_{1}}+C_{3}^{p_{2}}\left(T-t_{k}\right)^{\alpha_{1}} \epsilon^{p_{1}},
\end{aligned}
$$

if $k$ is sufficiently large; this contradicts (3.13). Thus this completes our proof.

Remark. Actually, the vector $\left(\alpha_{1}, \alpha_{2}\right)$ satisfies

$$
(P-I)\left(\begin{array}{c}
\alpha_{1} \\
\alpha_{2}
\end{array}\right)=\left(\begin{array}{l}
-1 \\
-1
\end{array}\right) \text {. }
$$

\section{ACKNOWLEDGEMENT}

The author would like to thank the referee for his helpful comments. The author also would like to thank Dr. Vatsala and Dr. Deng for their discussion and references.

\section{REFERENCES}

[1] Deng, K., Blow-up rates for parabolic systems, Z. Angew Math. Phys., 46, 110-118 (1995).

[2] Escobedo, M. and Herrero, M. A., Boundedness and blowup for a semilinear reaction-diffusion system, J. Diff. Equ., 89, 176-202 (1991).

[3] Escobedo, M. and Levine, H.A., Critical blowup and global existence numbers for a weakly coupled system of reaction-differential equations, Arch. Rational Mech. Anal. 129, 47-100 (1995). MR 96d:35063

[4] Friedman, A. and Giga, Y., A single point blow-up for solutions of semilinear parabolic systems, J. Fac. Sci. Univ. Tokyo Sec. IA Math. 34, 65-79 (1987). MR 89b:35066

[5] Friedman, A and Mcleod, B., Blow-up of positive solutions of semilinear heat equations, Indiana Univ. Math. J. 34, 425-447 (1985). MR 86j:35089

[6] Galaktionov, V. A., Kurdyumov, S. P., and Samarskii, A. A., A parabolic system of quasilinear equations, I Differential Equations 21, 1049-1062 (1985).

[7] Galaktionov, V. A., Kurdyumov, S. P., and Samarskii, A. A., A parabolic system of quasilinear equations. II Differential Equations 19, 1558-1571 (1983). 
[8] Hu, B. and Yin, H.-M., The profile near blowup time for the solution of the heat equation with a nonlinear boundary condition, Trans. Amer. Math. Soc., 346, 117-135 (1995). MR 95c:35040

[9] Pao, C.-V., Nonlinear Parabolic and Elliptic Equations, Plenum Press, New York 1992.

[10] Rossi J., The blow-up rate for a system of heat equation with non-trivial coupling at the boundary, Math. Meth. Appl. Sci. 20, 1-11 (1997). MR 97k:35099

[11] Samarskii, A. A., Galaktionov, V. A., Kurdyumov, S. P., and Mikhailov, A. P., Blow-up in Quasilinear Parabolic Equations, Walter de Gruyter, Berlin, 1995. MR 96b:35003

[12] Wang L., The blow-up for a semilinear parabolic system. Mathematica Applicata (complement), 104-106 (1995).

[13] Zhang, K., On the blow-up rate of solution of semilinear parabolic equations system, J. Math. Study 27, No. 2, 102-108(1994).

[14] Zhang, K., Blow-up phenomena in solutions of systems of semilinear parabolic equations, J. Math. Res. Exposition 15, No. 1, 83-90(1995). MR 96b:35102

Department of Mathematics, University of Louisiana at Lafayette, Lafayette, LouISIANA 70504

E-mail address: 1xw0340@usl.edu

Current address: Department of Computer Science, University of Louisiana at Lafayette, Lafayette, Louisiana 70504

E-mail address: 1xw0340@usl.edu 